\title{
AN ELEMENTARY RENEWAL THEOREM FOR RANDOM COMPACT CONVEX SETS
}

\author{
ILYA S. MOLCHANOV,* CWI, Amsterdam \\ EDWARD OMEY,** EHSAL, Brussels \\ EUGENE KOZAROVITZKY,*** Niiravtoprom, Kiev
}

\begin{abstract}
A set-valued analog of the elementary renewal theorem for Minkowski sums of random closed sets is considered. The corresponding renewal function is defined as

$$
H(K)=\sum_{n=0}^{\infty} \boldsymbol{P}\left\{S_{n} \subset K\right\},
$$

where $S_{n}=A_{1} \oplus \cdots \oplus A_{n}$ are Minkowski (element-wise) sums of i.i.d. random compact convex sets. In this paper we determine the limit of $H(t K) / t$ as $t$ tends to infinity. For $K$ containing the origin as an interior point,

$$
\lim _{t \rightarrow \infty} \frac{H(t K)}{t}=\inf _{u \in S_{A}^{+}} \frac{h_{K}(u)}{E h_{A}(u)},
$$

where $h_{K}(u)$ is the support function of $K$ and $S_{A}^{+}$is the set of all unit vectors $u$ with $E h_{A}(u)>0$. Other set-valued generalizations of the renewal function are also suggested.

AUMANN EXPECTATION; MINKOWSKI ADDITION; RENEWAL FUNCTION

AMS 1991 SUBJECT CLASSIFICATION: PRIMARY 60D05
\end{abstract}

SECONDARY 52A22, 60K05

\section{Introduction}

This paper continues earlier papers by different authors; see Artstein and Vitale (1975), Cressie (1979), Lyashenko (1982), Weil (1982), Gine et al. (1983), Puri and Ralescu (1985), etc. Their aims were to prove analogs of classical probability results (law of large numbers, central limit theorems, law of the iterated logarithm) for Minkowski sums of random closed sets.

Let $A$ be a random closed set in the Euclidean space $\mathbb{R}^{d}$, i.e. $A$ is a random element with values in the family $\mathscr{F}$ of all closed subsets of $\mathbb{R}^{d}$ and measurable with respect to the $\sigma$-algebra generated by the classes $\{F \in \mathscr{F}: F \cap K \neq \varnothing\}$ for $K$ running through the family $\mathscr{K}$ of all compacts, see Matheron (1975). The random set $A$ is said to be compact (convex) if almost all its realizations are compact (convex) sets.

Received 17 August 1993; revision received 7 September 1994.

* Present address: Department of Statistics, University of Glasgow, Glasgow, G12 8QQ, UK.

Supported in part by the Alexander von Humboldt-Stiftung, Bonn, Germany.

** Postal address: EHSAL, Stormstraat 2, 1000 Brussels, Belgium.

*** Postal address: Niiravtoprom, Dept. PMO, Predslavinskaja 28, 252145 Kiev, Ukraine. 
The Minkowski sum of two sets is defined elementwise:

$$
F_{1} \oplus F_{2}=\left\{x_{1}+x_{2}: x_{1} \in F_{1}, x_{2} \in F_{2}\right\} .
$$

Equipped with the operation $\oplus$, the family $\mathscr{F}$ is a semigroup. Furthermore, $\|F\|=\sup \{\|x\|: x \in F\}$ denotes the norm of $F$.

The law of large numbers for random sets in its simplest form (see Artstein and Vitale (1975)) states that, for any random compact set $A$ with $\boldsymbol{E}\|A\|<\infty$ and $A, A_{1}, \cdots, A_{n}$ i.i.d.,

$$
\rho_{\mathrm{H}}\left(n^{-1}\left(A_{1} \oplus \cdots \oplus A_{n}\right), \boldsymbol{E} A\right) \rightarrow 0 \quad \text { a.s. } \quad \text { as } \quad n \rightarrow \infty .
$$

Here $\rho_{\mathrm{H}}$ is the Hausdorff distance, namely

$$
\rho_{\mathrm{H}}\left(K, K_{1}\right)=\inf \left\{r>0: K \subset K_{1}^{r}, K_{1} \subset K^{r}\right\}
$$

where $K^{r}=K \oplus B_{r}(0), B_{r}(x)$ is the ball of radius $r$ centered at $x$. The set $\boldsymbol{E} A$ is the Aumann expectation of $A$, i.e. the convex set having support function

$$
h_{E A}(u)=E h_{A}(u), \quad u \in \mathbb{S}^{d-1},
$$

see Vitale (1988). Here $\mathbb{S}^{d-1}$ is the unit sphere in $\mathbb{R}^{d}$ and $h_{A}($.$) is the support$ function defined as

$$
h_{A}(u)=\sup \{\langle u, x\rangle: x \in A\},
$$

where $\langle u, x\rangle$ is the scalar product of $u$ and $x$.

Later on Cressie (1979), Lyashenko (1982) and Weil (1982) established the central limit theorem for Minkowski sums of random closed sets. The main difficulty in its formulation is caused by the fact that Minkowski sums of sets cannot be centered, since $(\mathscr{F}, \oplus)$ is a semigroup only. On the other hand, a random closed set with zero expectation coincides with the origin $o$ almost surely. The limit theorem of Weil (1982) states that if $\boldsymbol{E}\|A\|^{2}<\infty$, then

$$
n^{1 / 2} \rho_{\mathrm{H}}\left(n^{-1}\left(A_{1} \oplus \cdots \oplus A_{n}\right), \boldsymbol{E} A\right) \stackrel{\mathrm{d}}{\rightarrow} \sup _{u \in \mathbb{S}^{d-1}}|\zeta(u)| \quad \text { as } n \rightarrow \infty,
$$

where $\zeta$ is a continuous centered Gaussian process on the unit sphere, such that

$$
\boldsymbol{E} \zeta(u) \zeta(v)=E h_{A}(u) h_{A}(v)-h_{E A}(u) h_{E A}(v) .
$$

If the random function $\zeta$ is the support function of a certain random closed set $Z$, then $Z$ is said to be Gaussian. It admits the representation $Z=\xi+M$ for a Gaussian vector $\xi$ and non-random convex $M$, see Lyashenko (1983), Vitale (1984). Similar results for random closed sets in Banach spaces were proved by Gine et al. (1983) and Puri and Ralescu (1985).

Note that the Minkowski addition of sets corresponds to the addition of their support functions. Therefore, the strong law of large numbers and the central limit theorem for Minkowski sums of random sets can be proved by invoking the 
corresponding results for Banach spaces. On the other hand, Banach space variants of renewal theorems are not known up to now.

\section{Multidimensional renewal theorem}

The aim of this paper is to prove a random set analog of the elementary renewal theorem. This theorem states that

$$
H(t) / t \rightarrow 1 / E \xi_{1} \quad \text { as } t \rightarrow \infty,
$$

where

$$
H(t)=\sum_{n=0}^{\infty} \boldsymbol{P}\left\{S_{n} \leqq t\right\}
$$

is the renewal function, $S_{n}=\xi_{1}+\cdots+\xi_{n}, n \geqq 1$, are partial sums of i.i.d. nonnegative random variables, $S_{0}=0$.

A two-dimensional vector analog of this theorem was established by Omey (1989), see also Hunter (1974) and Bickel and Yahav (1965). We give below its $d$-dimensional variant.

Let $\xi_{i}=\left(\xi_{i 1}, \cdots, \xi_{i d}\right), i \geqq 1$, be a sequence of i.i.d. random vectors with the distribution function $F\left(x_{1}, \cdots, x_{d}\right)=\boldsymbol{P}\left\{\xi_{11} \leqq x_{1}, \cdots, \xi_{1 d} \leqq x_{d}\right\}$, and let $S_{n}=\sum_{i=1}^{n} \xi_{i}$ denote the corresponding partial sums. Define the counting process

$$
N(x)=N\left(x_{1}, \cdots, x_{d}\right):=\operatorname{card}\left\{n: S_{n 1} \leqq x_{1}, \cdots, S_{n d} \leqq x_{d}\right\}
$$

and the renewal function

$$
\begin{aligned}
H(x)=H\left(x_{1}, \cdots, x_{d}\right): & =\boldsymbol{E}\left(N\left(x_{1}, \cdots, x_{d}\right)+1\right) \\
& =\sum_{n=0}^{\infty} \boldsymbol{P}\left\{S_{n 1} \leqq x_{1}, \cdots, S_{n d} \leqq x_{d}\right\} .
\end{aligned}
$$

Theorem 2.1. Assume that $\boldsymbol{E} \xi_{1 i}=\mu_{i}$ are positive and finite, $1 \leqq i \leqq d$. Furthermore, let $\boldsymbol{E}\left(\xi_{1 i}^{-}\right)^{2}<\infty, 1 \leqq i \leqq d$, where $\xi_{1 i}^{-}$is the negative part of $\xi_{1 i}$. Then, for all finite positive $x_{1}, \cdots, x_{d}$,

$$
\lim _{t \rightarrow \infty} \frac{1}{t} H\left(t x_{1}, \cdots, t x_{d}\right)=\min \left(\frac{x_{1}}{\mu_{1}}, \cdots, \frac{x_{d}}{\mu_{d}}\right) .
$$

If $\max \left(\mu_{i}\right)=\infty$, then the limit is equal to zero.

Proof. Note that the conditions of the theorem yield the finiteness of $H(x)$ for all $x \in \mathbb{R}^{d}$. Suppose that $\max \left(\mu_{i}\right)<\infty$. For each $a>0$ and each $i$ define $N_{i}(a)$ and $M(a)$ 
934•SGSA ILYA S. MOLCHANOV, EDWARD OMEY AND EUGENE KOZAROVITZKY

as follows:

$$
\begin{aligned}
& N_{i}(a)=\operatorname{card}\left\{n: S_{n i} \leqq a\right\}, \\
& M(a)=\operatorname{card}\left\{n:\left|S_{n}\right| \leqq a\right\},
\end{aligned}
$$

where $\left|S_{n}\right|=\max _{1 \leqq i \leqq d}\left|S_{n i}\right|$ denotes the max-norm. Clearly, we have

$$
M(a) \leqq N(a, \cdots, a) \leqq \min _{1 \leqq i \leqq d} N_{i}(a)
$$

for all $a>0$. Also, replacing $\xi_{k i}$ with $\xi_{k i} / x_{i}$ yields

$$
M\left(x_{1}, \cdots, x_{d}, t\right) \leqq N(x t) \leqq \min _{1 \leqq i \leqq d} N_{i}\left(t x_{i}\right),
$$

where

$$
M\left(x_{1}, \cdots, x_{d}, t\right)=\operatorname{card}\left\{n: \max _{1 \leqq i \leqq d}\left|S_{n i}\right| / x_{i} \leqq t\right\}
$$

It was shown by Lai (1975) that

$$
N_{i}\left(t x_{i}\right) / t \rightarrow x_{i} / \mu_{i} \quad \text { a.s. } \quad \text { as } t \rightarrow \infty,
$$

and

$$
\lim _{t \rightarrow \infty} E N_{i}\left(t x_{i}\right) / t=x_{i} / \mu_{i}
$$

Expression (2.4) holds if the mean is positive; expression (2.5) holds if the second moment of the negative part of $\xi_{1 i}$ is finite.

As to $M(x)$, we use the strong law of large numbers to obtain that

$$
\left|S_{n}\right| / n \rightarrow|\mu| \text { a.s. } \quad \text { as } n \rightarrow \infty,
$$

where $\mu=\left(\mu_{1}, \cdots, \mu_{d}\right)$. From here it follows that

$$
M(t) / t \rightarrow|\mu|^{-1} \text { a.s. } \quad \text { as } t \rightarrow \infty,
$$

and consequently that

$$
M\left(x_{1}, \cdots, x_{d}, t\right) / t \rightarrow|\mu / x|^{-1}=\min _{1 \leq i \leq d} x_{i} / \mu_{i} \quad \text { a.s. } \quad \text { as } t \rightarrow \infty .
$$

Combining (2.3), (2.4) and (2.6) yields

$$
N(t x) / t \rightarrow \min _{1 \geqq i \leqq d} x_{i} / \mu_{i} \quad \text { a.s. } \quad \text { as } t \rightarrow \infty .
$$


Using (2.5), (2.7) and Pratt's extension of Lebesgue's theorem (see for example Johns (1957)), we obtain that

$$
\lim _{t \rightarrow \infty} E N(t x) / t=\min _{1 \leqq i \leqq d} x_{i} / \mu_{i}
$$

Hence (2.2) follows. If $\max \left(\mu_{i}\right)=\infty$, then it is necessary to truncate the components of $\xi$ with a truncation bound going to infinity.

Corollary 2.2. Suppose that in Theorem 2.1 all $\mu_{i}$ are finite and that $\max \left(\mu_{i}\right)>0$. If $E\left(\xi_{1 i}^{-}\right)^{2}<\infty$, then, for all finite positive $x_{1}, \cdots, x_{d}$,

$$
\lim _{t \rightarrow \infty} \frac{1}{t} H\left(t x_{1}, \cdots, t x_{d}\right)=\min \left(\frac{x_{i}}{\mu_{i}}: 1 \leqq i \leqq d, \mu_{i}>0\right) .
$$

Proof. Without loss of generality suppose that only $\mu_{1}=\boldsymbol{E} \xi_{11} \leqq 0$ (note that $\mu_{1}>-\infty$ due to the condition $\left.\boldsymbol{E}\left(\xi_{1 i}^{-}\right)^{2}<\infty\right)$. Consider a new random vector $\tilde{\xi}_{1}=\left(\xi_{11}-a, \xi_{12}, \cdots, \xi_{1 d}\right)$ for $a<\mu_{1}$. Then $\tilde{\xi}_{1}$ satisfies the condition of Theorem 2.1 , that is

$$
\lim _{t \rightarrow \infty} \frac{1}{t} \tilde{H}\left(t x_{1}, \cdots, t x_{d}\right)=\min \left(\frac{x_{1}}{\mu_{1}-a}, \frac{x_{2}}{\mu_{2}}, \cdots, \frac{x_{d}}{\mu_{d}}\right),
$$

where $\tilde{H}$ is the renewal function constructed by the vector $\tilde{\xi}_{1}$ and (2.1). Furthermore,

$$
\lim _{t \rightarrow \infty} \frac{1}{t} \bar{H}\left(t x_{1}, \cdots, t x_{d}\right)=\min \left(\frac{x_{2}}{\mu_{2}}, \cdots, \frac{x_{d}}{\mu_{d}}\right),
$$

where $\bar{H}$ is the renewal function constructed by realizations of the random vector $\bar{\xi}_{1}=\left(0, \xi_{12}, \cdots, \xi_{1 d}\right)$. Evidently,

$$
\widetilde{H}(x) \leqq H(x) \leqq \bar{H}(x) .
$$

Now the statement can be easily deduced from (2.10) and (2.11) by letting $a \uparrow \mu_{1}$.

The elementary renewal theorem for almost surely positive random variables $(d=1)$ follows from the Blackwell theorem. The latter result was generalized for the multidimensional case in Nagaev (1979) and Gafurov (1980), see also Gut (1988).

\section{Renewal theorem for random sets}

In what follows we shall consider a renewal theorem for Minkowski sums of random compact convex sets in $\mathbb{R}^{d}$. Recall that the distribution of a general random closed set $A$ is determined uniquely by the so-called capacity (or hitting) functional

$$
T(K)=\boldsymbol{P}\{A \cap K \neq \varnothing\}, \quad K \in \mathscr{K},
$$

see Matheron (1975), Stoyan et al. (1987). It is also known (see Vitale (1983) and 
Molchanov (1983)) that the distribution of a random compact convex set is determined by the containment functional

$$
H(K)=\boldsymbol{P}\{A \subset K\}
$$

defined on the class $\mathscr{C}_{0}$ of convex compact sets.

Let $A, A_{1}, A_{2}, \cdots$ be i.i.d. random compact convex sets, and let

$$
S_{n}=A_{1} \oplus \cdots \oplus A_{n}
$$

be their partial sums $\left(S_{0}=\{o\}\right.$ is the origin). For a closed set $K$ define the containment renewal function

$$
H(K)=\sum_{n=0}^{\infty} \boldsymbol{P}\left\{S_{n} \subset K\right\} .
$$

If $d=1, K=[0, t]$, and $A=\{\xi\}$ for a non-negative random variable $\xi$, then $H(K)$ is the classical renewal function. Our aim is to find the limit of $H(t K) / t$ as $t \rightarrow \infty$.

The set-valued renewal theorem may be of use when exact locations of the summands (random variables or vectors) are not known. Then the random variable $\xi$ is replaced by the random set $A$ and the sum of random variables turns into the Minkowski sum (3.1).

The following theorem is the most simple of its kind. For any set $M \subset \mathbb{R}^{d}$ let int $M$ and conv $(M)$ denote the interior and the convex hull of $M$ respectively.

Theorem 3.1. Suppose that $\boldsymbol{E}\|A\|<\infty$ and $\boldsymbol{E} \rho(o, \operatorname{conv}(A))^{2}<\infty$, where $\rho(o, A)$ is the minimum distance between points of $A$ and the origin. Consider a convex compact $K$, such that $o \in$ int $K$. Then

$$
\lim _{t \rightarrow \infty} \frac{H(t K)}{t}=\inf _{u \in S_{A}^{+}} \frac{h_{K}(u)}{h_{E A}(u)},
$$

where $S_{A}^{+}=\left\{x \in \mathbb{S}^{d-1}: h_{E A}(u)>0\right\}$.

Proof. First, note that $S_{n} \subset t K$ iff the support function of $S_{n}$ is not greater than the support function of $t K$, that is

$$
h_{S_{n}}(u) \leqq t h_{K}(u), \quad u \in \mathbb{S}^{d-1} .
$$

The left-hand side is equal to $\sum_{i=1}^{n} h_{A_{1}}(u)$. Note also that

$$
\sup _{u \in \mathbb{S}^{d-1}} h_{A}(u)^{-}<\rho(o, \operatorname{conv}(A)),
$$

where $h_{A}(u)^{-}$is the negative part of the support function $h_{A}(u)$.

Choose an $\varepsilon$-net $u_{1}, \cdots, u_{m}$ on the unit sphere $\mathbb{S}^{d-1}$. Then

$$
\Gamma_{\varepsilon}^{\prime}=\bigcap_{j=1}^{m} l_{u}\left(x_{j}^{\prime}\right) \subset K \subset \Gamma_{\varepsilon}^{\prime \prime}=\bigcap_{j=1}^{m} l_{u,}\left(x_{j}^{\prime \prime}\right)
$$


for suitable positive reals $x_{j}^{\prime}, x_{j}^{\prime \prime}, 1 \leqq j \leqq m$. Here $l_{u}(x)=\left\{y \in \mathbb{R}^{d}:\langle y, u\rangle \leqq x\right\}$, i.e. $K$ can be approximated by polyhedrons with facets orthogonal to $u_{j}, 1 \leqq j \leqq m$, and

$$
\rho_{\mathrm{H}}\left(\Gamma_{\varepsilon}^{\prime}, \Gamma_{\varepsilon}^{\prime \prime}\right) \rightarrow 0 \quad \text { as } \quad \varepsilon \rightarrow 0
$$

Then, by (2.9),

$$
\begin{aligned}
\frac{H(t K)}{t} & \geqq t^{-1} \sum_{n=0}^{\infty} \boldsymbol{P}\left\{\sum_{i=1}^{n} h_{A_{i}}\left(u_{j}\right) \leqq t x_{j}^{\prime} ; 1 \leqq j \leqq m\right\} \\
& \rightarrow \min _{1 \leqq j \leqq m ; u_{j} \in S_{A}^{+}} \frac{x_{j}^{\prime}}{\boldsymbol{E} h_{A}\left(u_{j}\right)} \\
& \geqq \min _{1 \leqq j \leqq m ; u_{j} \in S_{A}^{+}} \frac{h_{K}\left(u_{j}\right)-\rho_{\mathrm{H}}\left(\Gamma_{\varepsilon}^{\prime}, K\right)}{\boldsymbol{E} h_{A}\left(u_{j}\right)} .
\end{aligned}
$$

A similar bound from above yields

$$
\min _{1 \leqq j \leqq m ; u_{j} \in S_{A}^{+}} \frac{h_{K}\left(u_{j}\right)-\rho_{\mathrm{H}}\left(\Gamma_{\varepsilon}^{\prime}, K\right)}{E h_{A}\left(u_{j}\right)} \leqq \lim _{t \rightarrow \infty} \frac{H(t K)}{t} \leqq \min _{1 \leqq j \leqq m ; u_{j} \in S_{A}^{+}} \frac{h_{K}\left(u_{j}\right)+\rho_{\mathrm{H}}\left(\Gamma_{\varepsilon}^{\prime \prime}, K\right)}{E h_{A}\left(u_{j}\right)} .
$$

The continuity of the support function and (3.4) finish the proof.

Corollary 3.2. If $K=B_{1}(o)$, then

$$
\lim _{t \rightarrow \infty} H(t K) / t=(\|\boldsymbol{E} A\|)^{-1} .
$$

Corollary 3.3. Suppose that $A$ is isotropic (i.e. its distribution is invariant under rotations). Then

$$
\lim _{t \rightarrow \infty} H(t K) / t=a^{-1} \sup \left\{r: B_{r}(o) \subset K\right\},
$$

where

$$
a=\frac{1}{d b_{d}} \boldsymbol{E}\left[\int_{\mathbb{S}^{d-1}} h_{A}(u) d u\right]
$$

and $b_{d}$ is the volume of the unit ball in $\mathbb{R}^{d}$.

Proof. Evidently, $E A$ is a ball centered at the origin. Its radius $a$ can be evaluated using the fact that the mean width of the expectation is equal to the expected mean width of the set in question, see Vitale (1988). Then the result follows from Theorem 3.1. Note that if $d=2$, then $E \int_{\mathbb{S}^{d-1}} h_{A}(u) d u$ is the mean perimeter of $A$.

In particular, if $A=B_{\xi}(\eta)$ is the ball with rotation invariant distribution of its center, then $a=\boldsymbol{E} \xi$, and

$$
\lim _{t \rightarrow \infty} H(t K) / t=(\boldsymbol{E} \xi)^{-1} \sup \left\{r: B_{r}(o) \subset K\right\} .
$$

The result does not depend on the distribution of $\eta$. 
Example 3.4. Let $A=\{\xi\}$ be a random singleton. If $\mathbb{E}\|\xi\|^{2}<\infty$, then Theorem 3.1 yields

$$
\lim _{t \rightarrow \infty} \frac{H(t K)}{t}=\sup \{r: r E \xi \in K\}=\frac{1}{g(K, E \xi)}
$$

where $g(K, x)=\inf \{r \geqq 0: x \in r K\}$ is the gauge function of $K$; see Schneider (1993), p. 43.

In the following the assumption $o \in \operatorname{int} K$ will be dropped. First, suppose that the convex set $K$ in Theorem 3.1 does not contain the origin.

Theorem 3.5. Suppose that $\boldsymbol{E}\|A\|<\infty$, and $\boldsymbol{E} \rho(o \text {, conv }(A))^{2}<\infty$. If $K$ is convex and $o \notin K$, then

$$
\lim _{t \rightarrow \infty} \frac{H(t K)}{t}=\alpha_{K}-\min \left(\alpha_{K}, \beta_{K}\right)
$$

where

$$
\begin{aligned}
& \alpha_{K}=\inf \left\{\frac{h_{K}(u)}{h_{E A}(u)}: u \in \mathbb{S}^{d-1} ; h_{K}(u)>0, h_{E A}(u)>0\right\}, \\
& \beta_{K}=\inf \left\{\frac{h_{K}(u)}{h_{E A}(u)}: u \in \mathbb{S}^{d-1} ; h_{K}(u)<0, h_{E A}(u)<0\right\} .
\end{aligned}
$$

Here $\inf \varnothing=\infty$ and $\infty-\infty=0$.

Proof. Similarly to the proof of Theorem 3.1 and using the same notation we get

$$
\begin{gathered}
\frac{H(t K)}{t} \geqq t^{-1} \sum_{n=0}^{\infty} \boldsymbol{P}\left\{\sum_{i=1}^{n} h_{A_{i}}\left(u_{j}\right) / x_{j}^{\prime} \leqq t: 1 \leqq j \leqq m, x_{j}^{\prime}>0\right\} \\
-t^{-1} \sum_{n=0}^{\infty} \boldsymbol{P}\left\{\sum_{i=1}^{n} h_{A_{i}}\left(u_{j}\right) / x_{j}^{\prime} \leqq t: 1 \leqq j \leqq m\right\} .
\end{gathered}
$$

Note that the approximation of $K$ can be chosen in such a way that all $x_{i}^{\prime}$ are non-vanishing. Therefore, the proof can be finished similarly to the proof of Theorem 3.1.

If $o \in A$ a.s., then $\beta_{K}=\infty$, whence $H(t K) / t$ tends to zero. Furthermore,

$$
H(t E A) / t \rightarrow\left\{\begin{array}{ll}
1 & \text { if } o \in \operatorname{Int} E A \\
0 & \text { if } o \notin E A
\end{array} \text { as } t \rightarrow \infty .\right.
$$

Im Theorems 3.1 and 3.5 it is easy to obtain a corollary for the renewal function

$$
H(K)=\sum_{n=0}^{\infty} \boldsymbol{P}\left\{S_{n} \in K\right\}
$$


of a random singleton $A=\{\xi\}$, since in this case $h_{E A}(u)=\langle u, E \xi\rangle$; see also Example 3.4 .

Similar results as in Theorems 3.1 and 3.5 can be obtained for $K$ being the finite union of disjoint convex compacts, since

$$
H\left(\bigcup_{i=1}^{m} K_{i}\right)=\sum_{i=1}^{m} H\left(K_{i}\right)
$$

Results for the case when the origin is a boundary point of $K$ can be deduced from Theorems 3.1 and 3.5. Denote

$$
S_{K}^{+}=\left\{u \in \mathbb{S}^{d-1}: h_{K}(u)>0\right\} .
$$

Furthermore, let $\mathbb{C}$ be the minimum cone containing $K$.

Theorem 3.6. Suppose that $\boldsymbol{E}\|A\|<\infty, \boldsymbol{E} \rho(o \text {, conv }(A))^{2}<\infty$, and the origin is a boundary point of a convex compact $K$. If $A \subset \mathbb{C}$ a.s., then

$$
\lim _{t \rightarrow \infty} \frac{H(t K)}{t}=\inf _{u \in S_{A}} \frac{h_{K}(u)}{h_{E A}(u)} .
$$

The same is valid if $S_{A}^{+} \subset S_{K}^{+}$and $o \notin E A$. If $S_{A}^{+} \not S_{K}^{+}$, then the limit in (3.8) is equal to zero.

Proof. For each $\delta>0$, the set $K^{\delta}$ satisfies the conditions of Theorem 3.1. Then

$$
\frac{H(t K)}{t} \leqq \frac{H\left(t K^{\delta}\right)}{t} \rightarrow \inf _{u \in S_{A}^{+}} \frac{h_{K}(u)+\delta}{h_{E A}(u)} \quad \text { as } s \rightarrow \infty .
$$

If $h_{0}=h_{E A}\left(u_{0}\right)>0$ for some $u_{0} \notin S_{K}^{+}$, then the limit is less than $\delta / h_{0}$. This proves the last assertion of the theorem.

It follows from (3.9) that the limit in (3.8) is less than the corresponding right-hand side. Let us suppose that $S_{A}^{+} \subset S_{K}^{+}$and $o \notin E A$. Pick $u_{0} \in($ int $\mathbb{C}) \cap \mathbb{S}^{d-1}$ and put $K_{\delta}=\left\{x \in K:\left\langle x, u_{0}\right\rangle \geqq \delta\right\}$. Since $o \notin K_{\delta}$, Theorem 3.5 is applicable with

$$
\alpha_{K_{\delta}} \rightarrow \inf _{u \in S_{A}^{+}} h_{K}(u) / h_{E A}(u) \quad \text { as } \delta \rightarrow 0
$$

and

$$
\beta_{K_{\delta}} \leqq \rho\left(o, K_{\delta}\right) / \rho(o, E A) \rightarrow 0 \quad \text { as } \delta \rightarrow 0 .
$$

Then (3.8) follows from (3.9) and (3.5).

If $A \subset \mathbb{C}$ a.s., then $S_{A}^{+} \subset S_{K}^{+}$and either $o \notin E A$ or $o \in A$ with probability 1 . The first case has been already considered. If $o \in A$ a.s., then $h_{A}(u) \geqq 0$ for all $u \in \mathbb{S}^{d-1}$, and the proof can be finished exactly like the proof of Theorem 3.1 using approximations $\Gamma_{\varepsilon}^{\prime}$ of $K$ from below such that $o \in \Gamma_{\varepsilon}^{\prime}$ for all $\varepsilon$. 


\section{Another variant of the renewal function in the set-valued case}

Let us define the inclusion renewal function

$$
I(K)=\sum_{n=0}^{\infty} \boldsymbol{P}\left\{K \subset S_{n}\right\}
$$

for a convex compact set $K$. If $o \in K$, then $I(t K)$ decreases, whence either $I(t K) / t \rightarrow 0$ as $t \rightarrow \infty$ or $I(K)=\infty$. Thus we have to consider only the case $o \notin K$. The following result is proved similarly to Theorem 3.5 .

Theorem 4.1. Under the conditions and with the notation of Theorem' 3.5

$$
\lim _{t \rightarrow \infty} \frac{I(t K)}{t}=\beta_{K}-\min \left(\alpha_{K}, \beta_{K}\right),
$$

if $o \notin \boldsymbol{E} A$. If $o \in \operatorname{int} \boldsymbol{E} A$, then $I(K)$ is infinite. If $o$ belongs to the boundary of $\boldsymbol{E} A$, then the limit of $I(t K) / t$ is equal to zero in case $h_{K}(u) \neq 0$ and $h_{E A}(u)=0$ for some $u$. Otherwise $I(K)$ is infinite.

\section{Concluding remarks}

1. It is interesting also to consider the hitting renewal function

$$
U(K)=\sum_{n=0}^{\infty} \boldsymbol{P}\left\{S_{n} \cap K \neq \varnothing\right\} .
$$

In this case the situation is more complicated. Clearly, if $A$ is a singleton, then $U$ coincides with the containment renewal function $H$. Similarly, for a one-point compact $K$ Theorem 4.1 can be applied.

If $A=[\xi, \eta]$ is a convex subset of the line $(d=1)$, then a renewal theorem for the hitting function easy follows from Theorem 2.1. It is easily seen that

$$
\boldsymbol{P}\{A \cap[a, b] \neq \varnothing\}=\boldsymbol{P}\{\xi \leqq a, \eta \geqq a\}+\boldsymbol{P}\{\xi \in(a, b]\} .
$$

First, suppose that $a, b>0$, and $\boldsymbol{E} \xi, \boldsymbol{E} \eta>0$. Then

$$
\lim _{t \rightarrow \infty} U(t[a, b]) / t=b / \boldsymbol{E} \xi-\min (b / \boldsymbol{E} \eta, a / \boldsymbol{E} \xi) .
$$

Similar results can be obtained for other $a, b$. Let $a<0, b>0$. If $E \xi<0$ and $\boldsymbol{E} \eta>0$, then $U([a, b])$ is infinite. If both $\boldsymbol{E} \xi, \boldsymbol{E} \eta$ are positive, then

$$
\lim _{t \rightarrow \infty} U(t[a, b]) / t=b / \boldsymbol{E} \xi
$$

Moreover, further formulae can be obtained for $K$ being a finite union of disjoint segments.

2. It was mentioned in Smith (1964) that the finiteness of the second moment of 
the negative part of the random variable in Theorem 2.1 is necessary and sufficient for the finiteness of the corresponding renewal function.

If in Theorem 2.1 we assume that $\boldsymbol{E}\left(\xi_{1 i}^{-}\right)^{r}<\infty$ for $r>2$, then (2.2) can be replaced by

$$
\lim _{t \rightarrow \infty} \boldsymbol{E}\left(\frac{N(t x)}{t}\right)^{r}=\left(\min \left(\frac{x_{i}}{\mu_{i}}\right)\right)^{r}
$$

A similar result can be formulated for the containment renewal counting process $N(K)=\operatorname{card}\left\{n: S_{n} \subset K\right\}$.

3. The famous Boolean model in stochastic geometry (see Stoyan et al. (1987)) is defined to be the union of 'points' of the Poisson point process on the space $\mathscr{K}$ of compact sets. A general point process on $\mathscr{K}$ yields the so-called grain-germ model.

Similarly to the points of the renewal point process on the line, the sets $S_{n}, n \geqq 0$, from (3.1) can be viewed as 'points' of a renewal set-valued point process on $\mathscr{K}$. Then their union $\cup_{n=0}^{\infty} S_{n}$ serves as a model of a random set. This model can be applied to describe a kind of growth both of location and size, since with each new summand in (3.1) the result is shifted away (if $o \notin E A$ ) and grows simultaneously.

\section{References}

ARtstein, Z. ANd Vitale, R. A. (1975) A strong law of large numbers for random compact sets. Ann. Prob. 3, 879-882.

Aumann, R. J. (1965) Integrals of set-valued functions. J. Math. Anal. Appl. 12, 1-12.

Bickel, J. ANd Yahav, J. A. (1965) Renewal theory in the plane. Ann. Math. Stat. 36, 946-955.

CRESSIE, N. (1979) A central limit theorem for random sets. Z. Wahrscheinlichkeitsth. 49, 37-47.

Gafurov, M. U. (1980) On the first exit time of a multidimensional random walk from expanding sets. Sov. Math. Dokl. 22, 492-493.

GINE, E., HAHN, M. G. AND ZINN, J. (1983) Limit theorems for random sets: application of probability in Banach space results. In Lecture Notes in Mathematics 990, pp. 112-135. Springer-Verlag, Berlin. York.

Gut, A. (1988) Stopped Random Walks: Limit Theorems and Applications. Springer-Verlag, New

HUNTER, J. J. (1974) Renewal theory in two dimensions: basic results. Adv. Appl. Prob. 6, 379-391. JoHNs, M. V. (1957) Non-parametric empirical Bayes procedures. Ann. Math. Stat. 28, 649-669.

LAI, T. L. (1975) On uniform integrability in renewal theory. Bull. Inst. Math. Acad. Sinica 3, 99-105.

LYASHENKO, N. N. (1982) Limit theorems for sum of independent compact random subsets. J. Soviet Math. 20, 2187-2196. 76-92.

LYASHENKO, N. N. (1983) Statistics of random compacts in the Euclidean space. J. Soviet Math. 21,

Matheron, G. (1975) Random Sets and Integral Geometry. Wiley, New York.

Molchanov, I. S. (1983) A generalization of the Choquet theorem for random sets with a given class of realizations. Theor. Veroyatnost. i Matem. Statist. 28 86-93. English translation in: Theory Prob. Math. Statist. (1984) 28, 99-106.

Nagaev, A. V. (1979) Renewal theorems in $\mathbb{R}^{d}$. Theory Prob. Appl. 34, 572-581.

OMEY, E. (1989) Multivariate Regular Variation and Applications in Probability Theory. EHSAL,

Eclectica N 74, Brussel.

PURI, M. L. AND RAlEsCU, D. A. (1985) Limit theorems for random compact sets in Banach space.

Math. Proc. Camb. Phil. Soc. 97, 151-158.

SCHNEIDER, R. (1993) Convex Bodies: The Brunn-Minkowski Theory. Cambridge University Press. 


\section{2•SGSA ILYA S. MOLCHANOV, EDWARD OMEY AND EUGENE KOZAROVITZKY}

SMITH, W. R. (1964) On the elementary renewal theorem for non-identically distributed variables. Pacific J. Math. 14, 673-700.

Stoyan, D., Kendall, W. S. And Mecke, J. (1987) Stochastic Geometry and Its Applications. Akademie-Verlag, Berlin/Wiley, Chichester.

VITALE, R. A. (1983) Some developments in the theory of random sets. Bull. Int. Statist. Inst. 50, 863-871.

Vitale, R. A. (1984) On Gaussian random sets. In Stochastic Geometry, Geometric Statistics, Stereology, eds. R. V. Ambartzumian and W. Weil, pp. 222-224. Teubner, Stuttgart.

VITALE, R. A. (1988) An alternate formulation of mean value for random geometric figures. $J$. Microscopy 151, 197-204.

WeIL, W. (1982) An application of the central limit theorem for Banach-space-valued random variables to the theory of random sets. Z. Wahrscheinlichkeitsth. 60, 203-208. 